\title{
ENRICHISSEMENT DES INTERPOLATIONS D'ÉLÉMENTS FINIS EN UTILISANT DES MÉTHODES SANS MAILLAGE*
}

\author{
Antonio Huerta ${ }^{1}$, Sonia Fernández-Méndez ${ }^{1}$ et Pedro Díez ${ }^{1}$
}

\begin{abstract}
In the framework of meshless methods, the interpolation is based on a distribution of particles: it is not necessary to define connectivities. In these methods the interpolation can be easily enriched, increasing the number of particles (as in $h$-refinement of finite elements) or increasing the order of consistency (as in $p$-refinement of finite elements). However, comparing with finite elements, particle methods suffer from an increase in the computational cost, mainly due to the computation of the shape functions. In this paper, a mixed interpolation that combines finite elements and particles is presented. The objective is to take advantage of both methods. In order to define $h-p$ refinement strategies an a priori error estimate is needed, and thus, some convergence results are presented and proved for this mixed interpolation.
\end{abstract}

Résumé. Les méthodes sans maillage emploient une interpolation associée à un ensemble de particules : aucune information concernant la connectivité ne doit être fournie. Un des atouts de ces méthodes est que la discrétisation peut être enrichie d'une façon très simple, soit en augmentant le nombre de particules (analogue à la stratégie de raffinement $h$ ), soit en augmentant l'ordre de consistance (analogue à la stratégie de raffinement $p$ ). Néanmoins, le coût du calcul des fonctions d'interpolation est très élevé et ceci représente un inconvénient vis-à-vis des éléments finis. Cet article présente une interpolation mixte éléments finis-particules qui résulte de la généralisation de plusieurs travaux dans ce domaine. La formulation de cette interpolation mixte est valable pour n'importe quel ordre de consistance. Dans ce contexte, on énonce un estimateur d'erreur a priori dont la démonstration se base dans les propriétés de l'interpolation mixte. Ce résultat permet d'étudier la convergence de la méthode d'enrichissement et d'établir les stratégies de raffinement de l'interpolation qui permettent d'atteindre une solution avec une précision satisfaisante.

1991 Mathematics Subject Classification. 65N15, 65N50, 65N30.

Reçu le 24 octobre 2001. Révisé le 10 mai 2002.

\section{INTRODUCTION}

Les méthodes de particules ou méthodes sans maillage telles que RKPM [13,14], EFG [1-4,17,19], SPH [12,20] ou DEM [18] se sont avérées utiles en tant qu'instrument de calcul en mécanique. Ces méthodes n'ont pas besoin

\footnotetext{
Keywords and phrases. Adaptivité, raffinement $h-p$, éléments finis, méthodes sans maillage, méthodes particulaires, interpolation mixte, convergence.

* Financé partiellement par le Ministerio de Ciencia y Tecnología (DPI2001-2204).

1 Departamento de Matemática Aplicada III, Universidad Politécnica de Cataluña, Campus Norte UPC, 08034 Barcelona, Espagne. e-mail : antonio.huerta@upc.es, page web : http://www.upc.es/ma3/lacan.html
} 
d'un maillage. Le raffinement adaptatif et la prise en compte des discontinuités deviennent ainsi plus faciles (voir, par exemple, dans [4] le traitement des ruptures). Les fonctions d'interpolation employées permettent en outre de reproduire des fortes variations dans les gradients, l'effet des forces concentrées et des grandes déformations. Néanmoins, son coût élevé empêche l'utilisation de cette méthode dans un grand nombre de problèmes pratiques.

D'un autre coté, du point de vue pratique, la méthode classique des éléments finis est moins coûteuse, permet d'implémenter facilement les conditions limites essentielles sans avoir à employer les multiplicateurs de Lagrange et, surtout, elle peut être appliquée dans un grand nombre de problèmes et elle est appuyée par une très longue expérience d'utilisation. Malgré tout, le coût de la génération du maillage dans la méthode des éléments finis est loin d'être négligeable, d'autant plus si l'adaptivité et le remaillage s'avèrent nécessaires.

Le but de ce travail est de proposer et d'analyser une méthode mixte qui combine les éléments finis avec les méthodes sans maillage du type EFG. La présentation et les développements se font dans le contexte des problèmes elliptiques. Cette méthode mixte est présentée comme un outil pour faciliter les calculs adaptatifs. En partant d'une solution éléments finis dont la précision est insuffisante, on envisage d'ajouter des particules pour enrichir la discrétisation dans les zones où c'est nécessaire. Pour concevoir la stratégie adaptative, il faut bien connaître le comportement de la convergence de la méthode mixte. L'estimation a priori de l'erreur permettra de déduire quelle est la densité de particules qu'il faut ajouter en chaque zone du domaine. Les résultats de ce travail doivent donc permettre d'établir des critères d'enrichissement de la discrétisation (en ajoutant des particules) en fonction d'une estimation de l'erreur (a posteriori) de la solution éléments finis.

On trouve déjà dans la littérature des exemples de l'utilisation conjointe des deux méthodes dans un même calcul. Il s'agit, bien sur, de tirer profit des avantages des deux. Par exemple, Belytschko et al. [3] ont proposé d'utiliser des éléments finis près du contour avec conditions de type Dirichlet et du EFG ailleurs. Ceci permet d'imposer facilement (avec les éléments finis) les conditions limites Dirichlet. Dans ce type de couplage, on emploie une zone de transition entre la partie du domaine discrétisée par éléments finis et celle discrétisée par particules EFG. Dans cette zone, il y a une interpolation mixte construite à partir des nœuds des éléments finis et des particules du voisinage. L'idée fondamentale de cette interpolation est de remplacer des nœuds par des particules et d'établir des fonctions de transition linéaires. On est donc obligé d'employer une interface linéaire entre les éléments finis et les particules de la taille d'un élément. Dans le même ordre d'idées, on trouve le travail de Hegen [8] où les deux domaines sont unis grâce à des multiplicateurs de Lagrange.

En suivant cette idée, dans [11] on a proposé une formulation qui généralise le travail de Belytschko et al. [3] pour n'importe quel degré d'interpolation en éliminant les fonctions de transition et la restriction de remplacer des nœuds par des particules. On peut ainsi ajouter autant de particules que l'on veut et là où l'on veut, sans être conditionné par le maillage éléments finis adjacent. Tout ceci est fait sans perte de continuité, puisque les multiplicateurs de Lagrange ne sont pas employés pour coupler les domaines, et en conservant l'ordre de la méthode partout dans le domaine.

Une autre approche à l'utilisation conjointe des méthodes avec et sans maillage est celle de Liu et al. [16] qui ont essayé de proposer une méthodologie pour enrichir les maillages des éléments finis avec des méthodes sans maillage. L'idée de calculer avec la méthode des éléments finis, estimer l'erreur et, ensuite, enrichir l'interpolation là où il le faut sans avoir à remailler, est très attirante. Les méthodes de particules sont une alternative qui permet de mettre en œuvre cette idée. Il s'agit tout simplement d'ajouter des particules là où il faut enrichir l'interpolation en conservant ou en éliminant les nœuds du maillage d'origine.

Dans cet article, on présente un estimateur d'erreur a priori de la solution par éléments finis enrichie avec des méthodes EFG. Une approche similaire peut être envisagée avec n'importe quelle autre méthode de particules. On discute, à partir de la borne de l'erreur, le domaine d'application de la méthode et ses propriétés de convergence. 


\section{Quelques notions FOndamentales DeS MÉthodes SANS MAILlaGe}

L'objectif des méthodes sans maillage est d'obtenir une approximation fonctionnelle du type

$$
u(x) \simeq \sum_{i} u\left(x_{i}\right) N_{i}^{\rho}(x),
$$

à partir d'un nuage de points (les particules) $x_{i}$. Il est donc nécessaire de définir les fonctions d'interpolation (ou de forme) associées $N_{i}^{\rho}(x)$, dans le domaine $\Omega$ correspondant $\left(\Omega \subset \mathbb{R}^{n}\right)$.

Dans les méthodes RKPM (Reproducing Kernel Particle Methods $[13,14])$ les fonctions d'interpolation, $N_{i}^{\rho}(x)$, s'obtiennent avec une méthode de moindres carrés mobiles (Moving Least Squares [6]) qui permet d'avoir la consistance de l'ordre voulu. La méthode EFG (Element Free Galerkin $[1-4,17,19]$ ) peut être vue comme un cas particulier de ces méthodes [15].

Ce qui s'ensuit est une brève description de la méthode EFG. On désigne par $\phi(x)$ la fonction positive, paire et avec support compact que l'on emploie pour générer l'espace des fonctions d'interpolation. On définit alors la fonction corrigée avec le paramètre de dilatation, $\rho$ qui caractérise le support de $\phi_{\rho}(x)$,

$$
\phi_{\rho}(x):=\phi\left(\frac{x}{\rho}\right) \quad \text { et } \quad \phi_{\rho}\left(x-x_{i}\right)=\phi\left(\frac{x-x_{i}}{\rho}\right)
$$

qui est la fonction centrée en une particule $x_{i}$.

Dans le cadre de la méthode EFG la fonction de forme associée à une particule $x_{i}$ se définit en corrigeant $\phi_{\rho}\left(x-x_{i}\right)$,

$$
N_{i}^{\rho}(x)=\widetilde{\boldsymbol{\alpha}}(x)^{T} \mathbf{P}\left(x_{i}\right) \phi_{\rho}\left(x-x_{i}\right),
$$

où $\mathbf{P}(x)=\left\{p_{0}(x), p_{1}(x) \ldots, p_{l}(x)\right\}^{T}$ est une famille de polynômes incluant une base de l'espace des polynômes de degré inférieur ou égal à $m$. En une dimension $(n=1)$ il est courant que les polynômes $p_{i}(x)$ coïncident avec ceux de la base triviale, c'est-à-dire, $p_{i}(x)=x^{i}$. Le vecteur $\widetilde{\boldsymbol{\alpha}}(x)$ est choisi tel que l'expression (1) interpole exactement tous les polynômes dans $\mathbf{P}(x)$, i.e.

$$
\mathbf{P}(x)=\sum_{i} \mathbf{P}\left(x_{i}\right) N_{i}^{\rho}(x) .
$$

C'est donc grâce à l'équation précédente qu'on réussit à faire que l'interpolation introduite dans (1) ait la consistance d'ordre $m$. Si on utilise cette interpolation d'ordre $m$ dans le contexte d'une formulation de Galerkin pour résoudre une EDP on peut reproduire toutes les démonstrations des résultats de convergence déjà standard en éléments finis. Il est cependant habituel d'employer une forme équivalente à (2), pour définir les fonctions d'interpolation, soit

$$
N_{i}^{\rho}(x)=\boldsymbol{\alpha}(x)^{T} \mathbf{P}\left(\frac{x-x_{i}}{\rho}\right) \phi_{\rho}\left(x-x_{i}\right),
$$

et imposer la condition de consistance

$$
\mathbf{P}(0)=\sum_{i} \mathbf{P}\left(\frac{x-x_{i}}{\rho}\right) N_{i}^{\rho}(x),
$$

équivalente à (3) lorsque $\rho$ est constant. Ceci permet de déterminer $\boldsymbol{\alpha}(x)$ pour tout point $x$.

Dans [11] on discute les conditions que la distribution des particules doit vérifier pour que la détermination des fonctions d'interpolation, $N_{i}^{\rho}$, soit possible.

Remarque 1.1. L'équivalence entre les équations (2) et (4) est un résultat classique [5,21]. Le schéma de la démonstration est le suivant : 
- On remarque que, pour le choix trivial $\mathbf{P}(x)=\left[1, x, x^{2}, \ldots\right]^{\top}$, on a

$$
\mathbf{P}\left(\frac{x-x_{i}}{\rho}\right)=\mathbf{T}(x) \mathbf{P}\left(x_{i}\right),
$$

où $\mathbf{T}(x)$ est une matrice triangulaire avec des éléments dans la diagonale égaux à 1 ou -1 . Donc, cette matrice est inversible. Cette propriété se vérifie pour tout point $x_{i}$ du domaine, pas seulement pour les particules.

- Il suffit alors de remplacer $\widetilde{\boldsymbol{\alpha}}(x)$ par $\mathbf{T}(x) \boldsymbol{\alpha}(x)$ pour vérifier équivalence entre (2) et (4).

Ensuite, l'équivalence entre (3) et (5) se déduit en utilisant $\mathbf{T}(x) \mathbf{P}(x)=\mathbf{P}(0)$, qui est évident si on remplace $x_{i}$ par $x$ dans (6).

Remarque 1.2. Les conditions (3) et (5) sont équivalentes si et seulement si le paramètre de dilatation $\rho$ est constant. Si l'on veut utiliser un paramètre de dilatation qui varie en $x$, il convient d'employer l'expression suivante

$$
N_{i}^{\rho}(x)=\boldsymbol{\alpha}(x)^{T} \mathbf{P}\left(\frac{x-x_{i}}{\rho}\right) \phi\left(\frac{x-x_{i}}{\rho_{i}}\right),
$$

où $\rho_{i}$ est le paramètre de dilatation associé à la particule $x_{i}$ et $\rho$ se maintient constant dans $\mathbf{P}$. On remarquera que l'expression de (4) n'a pas été généralisée directement. La valeur constante $\rho$ est d'habitude une moyenne des valeurs des $\rho_{i}$. La condition de consistance dans ce cas sera (5), qui est encore équivalente à (3).

Remarque 1.3. Le paramètre de dilatation $\rho$ détermine le support des fonctions $N_{i}^{\rho}(x)$. Il est analogue à la taille des éléments $h$ dans une approche par éléments finis. Le raffinement équivalent au $h$ des éléments finis s'obtient en réduisant le paramètre de dilatation $\rho$ (ceci entraîne une augmentation de la densité des particules et donc de leur nombre). Liu et al. [15] ont démontré la convergence des méthodes RKPM et, en particulier de EFG. On obtient une borne d'erreur similaire à celle des éléments finis. Le paramètre $\rho$ joue le rôle de la taille d'élément $h$ et $m$ joue le rôle du degré d'interpolation $p$ dans l'approche éléments finis.

\section{Approche mixte hiÉrarchique D'EFG AVEC MEF}

On s'intéresse à interpoler une fonction $u$ dans un domaine $\Omega \subset \mathbb{R}^{n}$ en utilisant éléments finis et EFG. On considère ainsi un ensemble de nœuds $\left\{x_{i}\right\}_{i \in I^{h}}$ et leurs fonctions de forme associées $N_{i}^{h}(x)$. La partie de $u$ représentée par les éléments finis s'écrit :

$$
u^{h}(x)=\sum_{i \in I^{h}} u\left(x_{i}\right) N_{i}^{h}(x) .
$$

On considère en outre un ensemble de particules $\left\{x_{j}\right\}_{j \in I^{\rho}}$ et leurs fonctions d'interpolation associées $N_{j}^{\rho}(x)$ permettant de décrire la contribution de la méthode EFG à l'interpolation de $u$ :

$$
u^{\rho}(x)=\sum_{j \in I^{\rho}} u\left(x_{j}\right) N_{j}^{\rho}(x) .
$$

Dans le cas le plus général, le domaine $\Omega$ peut être exprimé comme l'union de deux sous domaines pas forcément disjoints (c'est-à-dire, avec la possibilité de chevauchements) :

$$
\Omega=\Omega^{\rho} \cup \Omega^{h}
$$

$\Omega^{h}$ est la région où les fonctions $N_{i}^{h}$ ont une influence dans l'approximation,

$$
\Omega^{h}=\left\{x \in \Omega / \exists i \in I^{h} \mid \quad N_{i}^{h}(x) \neq 0\right\},
$$


et $\Omega^{\rho}$ est la zone d'influence des fonctions $N_{j}^{\rho}$,

$$
\Omega^{\rho}=\left\{x \in \Omega / \exists j \in I^{\rho} \mid \quad N_{j}^{\rho}(x) \neq 0\right\}
$$

Dans la région où seulement les fonctions éléments finis agissent, $\Omega^{h} \backslash \Omega^{\rho}$, on considère une approximation éléments finis classique, donc consistante :

$$
u(x) \simeq u^{h}(x)
$$

Dans la région où il n'y a que les particules, $\Omega^{\rho} \backslash \Omega^{h}$, on considère une approximation EFG classique et donc encore consistante :

$$
u(x) \simeq u^{\rho}(x)
$$

Dans la zone commune où l'on trouve l'influence des nœuds et des particules,

$$
\widetilde{\Omega}:=\Omega^{h} \cap \Omega^{\rho},
$$

on considère une interpolation mixte :

$$
u(x) \simeq u^{h}(x)+u^{\rho}(x) .
$$

L'approche générale que l'on décrit en [11] permet d'inclure le couplage (compatibilité entre les domaines $\Omega^{h}$ et $\Omega^{\rho}$ différents) ainsi que l'enrichissement (existance de $\widetilde{\Omega}$ ). Néanmoins, le but de ce travail est d'étudier la convergence de la méthode d'enrichissement et, par conséquent, on va se centrer sur le domaine $\widetilde{\Omega}$. Par la suite on va supposer que l'enrichissement a lieu dans tout le domaine, c'est-à-dire que $\Omega$ coïncide avec $\widetilde{\Omega}$.

\subsection{Calcul des fonctions d'interpolation $\boldsymbol{N}_{j}^{\rho}$}

L'expression de l'interpolation mixte s'obtient en remplaçant (7) et (8) dans (9) :

$$
u(x) \simeq \sum_{i \in I^{h}} u\left(x_{i}\right) N_{i}^{h}(x)+\sum_{j \in I^{\rho}} u\left(x_{j}\right) N_{j}^{\rho}(x) .
$$

On rappelle que $N^{\rho}$ est définie par (4) de la même façon que dans la méthode EFG classique. Il faut ainsi déterminer le vecteur $\boldsymbol{\alpha}(x)$ à partir des conditions de consistance. La seule différence est que les conditions de consistance doivent être imposées maintenant sur l'interpolation donnée par (10). Il s'agit d'imposer que cette interpolation reproduise exactement les polynômes de degré inférieur ou égal à $m$. C'est-à-dire, il faut vérifier

$$
\mathbf{P}(0)=\sum_{j \in I^{\rho}} \mathbf{P}\left(\frac{x-x_{j}}{\rho}\right) N_{j}^{\rho}(x)+\sum_{i \in I^{h}} \mathbf{P}\left(\frac{x-x_{i}}{\rho}\right) N_{i}^{h}(x),
$$

qui est équivalent à (5). Si $\rho$ est constant dans tout le domaine, la condition de consistance (11) devient

$$
\mathbf{P}(x)=\sum_{j \in I^{\rho}} \mathbf{P}\left(x_{j}\right) N_{j}^{\rho}(x)+\sum_{i \in I^{h}} \mathbf{P}\left(x_{i}\right) N_{i}^{h}(x) .
$$

En remplaçant (4) dans (11) on obtient un système d'équations qui permet de déterminer $\boldsymbol{\alpha}$ :

$$
\mathbf{M}(x) \boldsymbol{\alpha}(x)=\mathbf{P}(0)-\sum_{i \in I^{h}} \mathbf{P}\left(\frac{x-x_{i}}{\rho}\right) N_{i}^{h}(x),
$$

avec

$$
\mathbf{M}(x)=\sum_{j \in I^{\rho}} \mathbf{P}\left(\frac{x-x_{j}}{\rho}\right) \mathbf{P}^{T}\left(\frac{x-x_{j}}{\rho}\right) \phi\left(\frac{x-x_{j}}{\rho}\right) .
$$


Ce système est très similaire à celui qui apparaît dans les méthodes EFG classiques. À la seule différence près que le terme de droite dans (13) doit être corrigé pour tenir compte de la présence des éléments finis.

Remarque 2.1. Les fonctions $N_{j}^{\rho}$ sont hiérarchiques. C'est-à-dire que les fonctions associées aux particules s'annulent dans tous les nœuds des éléments finis, autrement dit, $N_{j}^{\rho}\left(x_{k}\right)=0 \quad \forall j \in I^{\rho}, k \in I^{h}$. Ceci est facile à démontrer en remarquant que le terme de droite dans (13) devient nul pour $x=x_{k}, k \in I^{h}$ :

$$
\mathbf{P}(0)-\sum_{i \in I^{h}} \mathbf{P}\left(\frac{x_{k}-x_{i}}{\rho}\right) N_{i}^{h}\left(x_{k}\right)=\mathbf{P}(0)-\sum_{i \in I^{h}} \mathbf{P}\left(\frac{x_{k}-x_{i}}{\rho}\right) \delta_{i k}=\mathbf{0} .
$$

Il s'ensuit que la solution du système (13) est $\boldsymbol{\alpha}\left(x_{k}\right)=\mathbf{0}$ et que $N_{j}^{\rho}\left(x_{k}\right)=0$.

Il existe des restrictions qu'il faut vérifier lorsqu'il s'agit de placer les particules dans le domaine. Le nombre de particules doit être suffisant pour assurer que la matrice $\mathbf{M}(x)$ soit inversible dans tout point d'intégration. Ceci est discuté dans [11].

\section{AnAlyse De L'EnRIChissement Des ÉlÉments Finis AVEC EFG}

Dans cette section, on analyse la méthode qu'on vient d'introduire. Un résultat de convergence qui caractérise l'efficacité de l'approche mixte est énoncé et démontré. Dans la section 4 plusieurs tests numériques montrent que les bornes de l'erreur obtenues dans les résultats de convergence sont très précises.

\subsection{Résultats préalables}

La démonstration du théorème de convergence exige auparavant l'obtention de quelques résultats préalables. Ces résultats sont l'objet des lemmes qu'on inclut dans cette section.

Lemme 3.1. On suppose $\Omega$ ouvert et borné. Alors, la matrice $\mathbf{M}(x)$ introduite dans (14) pour tout $x$ dans $\Omega$ vérifie

$$
\left\|\mathbf{M}^{-1}(x)\right\|_{\infty} \leq C_{\mathbf{M}}
$$

où $C_{\mathbf{M}}$ est une constante indépendante de $\rho$ et $x$.

Démonstration. On se rapporte à un travail de Liu et al. [15] où ils démontrent que les coefficients de la matrice $\mathcal{M}$ définie par

$$
\mathcal{M}(x)=\int_{\Omega} \mathbf{P}\left(\frac{x-y}{\rho}\right) \mathbf{P}^{T}\left(\frac{x-y}{\rho}\right) \phi\left(\frac{x-y}{\rho}\right) \frac{1}{\rho^{n}} \mathrm{~d} y,
$$

sont bornés par une constante indépendante de $x$ et de $\rho$. Cette propriété peut s'exprimer comme «les coefficients de la matrice $\mathcal{M}$ sont d'ordre $1 »$. Ils remarquent ensuite que la matrice $\mathbf{M}$ définie dans (14) est différente de $\mathcal{M}$ définie dans (15) puisque les intégrales sont remplacées par des sommes. La matrice $\mathbf{M}$ définie dans (14) peut être vue comme une approximation de la matrice $\mathcal{M}$ de (15) en remplaçant les intégrales par des quadratures d'intégration numérique. Dans ce cas la quadrature emploie en tant que points d'intégration les particules $x_{j}$ «proches » de $x$ (telles que $\phi_{\rho}\left(x-x_{j}\right)$ soit non négligeable, c'est-à-dire dans un entourage de rayon $\rho$ autour de $x$ ) et en tant que poids d'intégration les valeurs

$$
\omega_{i}=\rho^{n} \propto \text { mesure }\left\{\operatorname{support}\left(\phi\left(\frac{x-x_{j}}{\rho}\right)\right)\right\} .
$$

Évidemment ceci introduit une erreur associée à la quadrature dans l'évaluation de $\mathbf{M}$ par rapport à l'expression « continue » de (15) qui est asymptotiquement négligeable. 
Lemme 3.2 (borne pour le terme indépendant du système). Soit $q_{r}(x):=x^{r}$. Alors, la composante $r$-ième du terme de droite du système (13) vérifie

$$
\left|q_{r}(0)-\sum_{i \in I^{h}} q_{r}\left(\frac{x-x_{i}}{\rho}\right) N_{i}^{h}(x)\right| \leq\left\{\begin{array}{cl}
0 & 0 \leq r \leq p \\
\left(\begin{array}{c}
r \\
p+1
\end{array}\right)\left[\frac{h}{\rho}\right]^{r} & p<r \leq m .
\end{array}\right.
$$

Démonstration. On introduit la notation

$$
t_{r}(x):=q_{r}(0)-\sum_{i \in I^{h}} q_{r}\left(\frac{x-x_{i}}{\rho}\right) N_{i}^{h}(x),
$$

pour la composante $r$-ième du terme de droite du système (13). Pour $r=1$ on a $q_{0}(x)=1$. Dans ce cas on a

$$
t_{0}(x)=1-\sum_{i \in I^{h}} 1 N_{i}^{h}(x)=0,
$$

car la base d'éléments finis interpole exactement les fonctions constantes. Pour $1 \leq r \leq m$ le terme de droite du système (13) s'écrit

$$
\begin{aligned}
t_{r}(x) & =0-\sum\left(\frac{x-x_{i}}{\rho}\right)^{r} N_{i}^{h}(x) \\
& =-\frac{1}{\rho^{r}} \sum_{i \in I^{h}}\left(x-x_{i}\right)^{r} N_{i}^{h}(x) \\
& =-\frac{1}{\rho^{r}} \sum_{i \in I^{h}}\left(\sum_{k=0}^{r}\left(\begin{array}{l}
r \\
k
\end{array}\right) x^{r-k}\left(-x_{i}\right)^{k}\right) N_{i}^{h}(x) \\
& =-\frac{1}{\rho^{r}} \sum_{k=0}^{r}\left[(-1)^{k}\left(\begin{array}{l}
r \\
k
\end{array}\right) x^{r-k}\left(\sum_{i \in I^{h}} x_{i}^{k} N_{i}^{h}(x)\right)\right]
\end{aligned}
$$

où $\sum x_{i}^{k} N_{i}^{h}(x)$ est l'approximation par éléments finis de la fonction $x^{k}$. Soit $E_{k}(x)$ l'erreur associée à cette approximation, c'est-à-dire

$$
\sum_{i \in I^{h}} x_{i}^{k} N_{i}^{h}(x)=x^{k}-E_{k}(x) .
$$

Si $k \leq p$ la base d'éléments finis reproduit exactement $x^{k}$. Donc

$$
E_{k}(x)=0, \quad k \leq p
$$

Si $k>p$, alors, d'après la formule d'erreur de Lagrange à l'intérieur de l'élément où se trouve $x$, on obtient

$$
E_{k}(x)=\left(\begin{array}{c}
k \\
p+1
\end{array}\right) \xi^{k-(p+1)} L(x), \quad k>p
$$

où $\xi=\xi(x)$ est un point appartenant à l'élément qui contient $x$ et

$$
L(x)=\left(x-x_{i_{0}}\right)\left(x-x_{i_{1}}\right) \ldots\left(x-x_{i_{p}}\right),
$$

est un polynôme associé aux nœuds de l'élément $x_{i_{j}}, j=0, \ldots, p$. 
En remplaçant (17) dans (16) on obtient

$$
\begin{aligned}
t_{r}(x) & =-\frac{1}{\rho^{r}} \sum_{k=0}^{r}\left[(-1)^{k}\left(\begin{array}{l}
r \\
k
\end{array}\right) x^{r-k}\left(x^{k}-E_{k}(x)\right)\right] \\
& =-\frac{1}{\rho^{r}} \sum_{k=0}^{r}\left[\left(\begin{array}{l}
r \\
k
\end{array}\right) x^{r-k}(-x)^{k}\right]+\frac{1}{\rho^{r}} \sum_{k=0}^{r}\left[(-1)^{k}\left(\begin{array}{l}
r \\
k
\end{array}\right) x^{r-k} E_{k}(x)\right] \\
& =-\frac{1}{\rho^{r}}(x-x)^{r}+\frac{1}{\rho^{r}} \sum_{k=0}^{r}\left[(-1)^{k}\left(\begin{array}{l}
r \\
k
\end{array}\right) x^{r-k} E_{k}(x)\right] \\
& =\frac{1}{\rho^{r}} \sum_{k=0}^{r}\left[(-1)^{k}\left(\begin{array}{l}
r \\
k
\end{array}\right) x^{r-k} E_{k}(x)\right] .
\end{aligned}
$$

Si $r \leq p$ (et puisque $k \leq r$ ) on peut employer (18) dans (21) et on obtient

$$
t_{r}(x)=0, \quad r \leq p,
$$

comme on voulait le démontrer. Si, au contraire, $r>p$, on emploie encore (18) et on remplace (19) dans (21) pour $k>p$. On obtient ainsi pour $r>p$

$$
t_{r}(x)=\frac{1}{\rho^{r}} \sum_{k=p+1}^{r}\left[(-1)^{k}\left(\begin{array}{l}
r \\
k
\end{array}\right) x^{r-k}\left(\begin{array}{c}
k \\
p+1
\end{array}\right) \xi^{k-(p+1)} L(x)\right] .
$$

En introduisant le changement de variable $j:=k-(p+1)$, on obtient

$$
t_{r}(x)=\frac{1}{\rho^{r}} \sum_{j=0}^{n}\left[(-1)^{j+(p+1)}\left(\begin{array}{c}
r \\
j+(p+1)
\end{array}\right) x^{n-j}\left(\begin{array}{c}
j+(p+1) \\
p+1
\end{array}\right) \xi^{j} L(x)\right]
$$

où

$$
n:=r-(p+1)
$$

Si on remarque que

$$
\left(\begin{array}{c}
r \\
j+(p+1)
\end{array}\right)\left(\begin{array}{c}
j+(p+1) \\
p+1
\end{array}\right)=\left(\begin{array}{c}
r \\
p+1
\end{array}\right)\left(\begin{array}{l}
n \\
j
\end{array}\right)
$$

l'expression pour $t_{r}(x)$ devient

$$
t_{r}(x)=\frac{(-1)^{(p+1)}}{\rho^{r}}\left(\begin{array}{c}
r \\
p+1
\end{array}\right) L(x) \sum_{j=0}^{n}\left[\left(\begin{array}{c}
n \\
j
\end{array}\right) x^{n-j}(-\xi)^{j}\right] .
$$

Il s'ensuit que

$$
t_{r}(x)=\frac{(-1)^{(p+1)}}{\rho^{r}}\left(\begin{array}{c}
r \\
p+1
\end{array}\right) L(x)(x-\xi)^{n} .
$$

Or, $|L(x)| \leq h^{p+1}$ et $|x-\xi| \leq h$ puisque $x$ et $\xi$ appartiennent au même élément. On retrouve donc la thèse du théorème

$$
\left|t_{r}(x)\right| \leq \frac{1}{\rho^{r}}\left(\begin{array}{c}
r \\
p+1
\end{array}\right) h^{n+(p+1)}=\left(\begin{array}{c}
r \\
p+1
\end{array}\right)\left[\frac{h}{\rho}\right]^{r} .
$$


Lemme 3.3. On suppose que $h<Q \rho$, avec

$$
Q=\min _{p+1 \leq r \leq m}\left(\begin{array}{c}
r \\
p+1
\end{array}\right)^{\frac{-1}{r-(p+1)}} .
$$

Alors, le terme de droite du système (13) vérifie

$$
\left\|\mathbf{P}(0)-\sum_{i \in I^{h}} \mathbf{P}\left(\frac{x-x_{i}}{\rho}\right) N_{i}^{h}(x)\right\|_{\infty} \leq\left[\frac{h}{\rho}\right]^{p+1} .
$$

Démonstration. L'hypothèse $(h<Q \rho)$ et la définition $(29)$ conduisent à l'inégalité

$$
\left(\begin{array}{c}
r \\
p+1
\end{array}\right)\left[\frac{h}{\rho}\right]^{r-(p+1)} \leq 1
$$

En employant le résultat énoncé dans le Lemme 3.2 , la composante $r$-ième du terme de droite de $t_{r}(x)$ du système (13) (pour $p+1 \leq r \leq m$ ) vérifie

$$
\left|t_{r}(x)\right| \leq \underbrace{\left(\begin{array}{c}
r \\
p+1
\end{array}\right)\left[\frac{h}{\rho}\right]^{r-(p+1)}}_{\leq 1} \frac{h^{p+1}}{\rho^{p+1}} \leq\left[\frac{h}{\rho}\right]^{p+1},
$$

comme il fallait le démontrer.

Lemme 3.4 (borne des fonctions d'interpolation). On suppose que $h$ est « assez petit » par rapport à $\rho$, c'est-à-dire $h<Q \rho$, avec $Q$ définie dans (29). Alors,

$$
\left|N_{j}^{\rho}(x)\right| \leq C\left[\frac{h}{\rho}\right]^{p+1}
$$

où $C$ est une constante indépendante de $h, \rho$ et $x$.

Démonstration. Le résultat est obtenu en employant les Lemmes 3.1 et 3.3 et en rappelant que si le point $x$ est sous le domaine d'influence de la particule $x_{j}$ (la fonction d'interpolation associée à $x_{j}$ ne s'annule pas dans $x$ ), alors $\left|x-x_{j}\right| / \rho \leq 1$. Il s'en déduit que, dans ces conditions

$$
\left\|\mathbf{P}\left(\frac{x-x_{j}}{\rho}\right)\right\|_{\infty} \leq 1
$$

et il s'ensuit que

$$
\begin{aligned}
\left|N_{j}^{\rho}(x)\right| & \leq\left\|\mathbf{P}\left(\frac{x-x_{j}}{\rho}\right)\right\|_{\infty}\left\|\mathbf{M}^{-1}(x)\right\|_{\infty}\left\|\mathbf{P}(0)-\sum_{i \in I^{h}} \mathbf{P}\left(\frac{x-x_{i}}{\rho}\right) N_{i}^{h}(x)\right\|_{\infty}\left|\phi\left(\frac{x-x_{j}}{\rho}\right)\right| \\
& \leq C\left[\frac{h}{\rho}\right]^{p+1} .
\end{aligned}
$$




\subsection{Théorème de convergence}

Théorème 3.1. On suppose $h<Q \rho$ avec $Q$ définie dans (29). On suppose aussi que l'inconnue u appartient $\grave{a} \mathrm{C}^{m+1}(\bar{\Omega})$, où $\Omega$ est un ouvert borné et que les fonctions $N_{j}^{R}$ sont celles qu'on a introduites dans la section précédente. Alors,

$$
\left\|u-\left(u^{h}+u^{R}\right)\right\|_{L_{\infty}} \leq h^{p+1}\left(C_{1} h^{q}+C_{2} \rho^{q}\right)
$$

où $C_{1}$ et $C_{2}$ sont indépendantes de $\rho$ et $h$.

Démonstration. Soit le développement en série de Taylor de $u$ autour de $x$ tronqué au terme $m$-ième

$$
\begin{aligned}
u\left(x_{i}\right)= & u(x)+\frac{\mathrm{d} u(x)}{\mathrm{d} x}\left(x_{i}-x\right)+\frac{1}{2} \frac{\mathrm{d}^{2} u(x)}{\mathrm{d} x^{2}}\left(x_{i}-x\right)^{2}+\ldots \\
& +\frac{1}{m !} \frac{\mathrm{d}^{m} u(x)}{\mathrm{d} x^{m}}\left(x_{i}-x\right)^{m}+\frac{1}{(m+1) !} \frac{\mathrm{d}^{m+1} u(\xi)}{\mathrm{d} x^{m+1}}\left(x_{i}-x\right)^{m+1},
\end{aligned}
$$

où $\xi$ est un point entre $x$ et $x_{i}$. On unifie la notation des fonctions d'interpolation en introduisant la définition suivante

$$
N_{i}(x):= \begin{cases}N_{i}^{h}(x) & i \in I^{h} \\ N_{i}^{\rho}(x) & i \in I^{\rho} .\end{cases}
$$

En remplaçant dans (31) l'expression de la fonction approchée,

$$
u(x) \simeq u^{\rho}(x)+u^{h}(x)=\sum_{i \in I^{h}} u\left(x_{i}\right) N_{i}^{h}(x)+\sum_{j \in I^{\rho}} u\left(x_{j}\right) N_{j}^{\rho}(x)=\sum_{i \in I^{h} \cup I^{\rho}} u\left(x_{i}\right) N_{i}(x),
$$

on obtient

$$
\begin{aligned}
u^{\rho}(x)+u^{h}(x)= & u(x) \underbrace{\sum_{i \in I^{h} \cup I^{\rho}} N_{i}(x)}_{1}+\frac{\mathrm{d} u(x)}{\mathrm{d} x} \underbrace{\sum_{i \in I^{h} \cup I^{\rho}}\left(x_{i}-x\right) N_{i}(x)}_{0} \\
& +\frac{1}{2} \frac{\mathrm{d}^{2} u(x)}{\mathrm{d} x^{2}} \underbrace{\sum_{i \in I^{h} \cup I^{\rho}}\left(x_{i}-x\right)^{2}}_{0} N_{i}(x)+\cdots+\frac{1}{m !} \frac{\mathrm{d}^{m} u(x)}{\mathrm{d} x^{m}} \underbrace{\sum_{i \in I^{h} \cup I^{\rho}}\left(x_{i}-x\right)^{m} N_{i}(x)}_{0} \\
& +\frac{1}{(m+1) !} \frac{\mathrm{d}^{m+1} u(\xi)}{\mathrm{d} x^{m+1}} \sum_{i \in I^{h} \cup I^{\rho}}\left(x_{i}-x\right)^{(m+1)} N_{i}(x) \\
= & u(x)+\frac{1}{(m+1) !} \frac{\mathrm{d}^{m+1} u(\xi)}{\mathrm{d} x^{m+1}} \sum_{i \in I^{h} \cup I^{\rho}}\left(x_{i}-x\right)^{(m+1)} N_{i}(x) .
\end{aligned}
$$

Dans l'expression précédente, on a employé la condition de consistance d'ordre $m$ exprimée par (5) des fonctions d'interpolation $N_{i}$ puisque les fonctions d'interpolation associées aux particules ont été construites de façon à que ceci soit vérifié. En réécrivant (31) :

$$
u(x)-\left(u^{\rho}+u^{h}\right)(x)=-\frac{1}{(m+1) !} \frac{\mathrm{d}^{m+1} u(\xi)}{\mathrm{d} x^{m+1}} \times\left(\sum_{i \in I^{h}}\left(x_{i}-x\right)^{m+1} N_{i}^{h}(x)+\sum_{j \in I^{\rho}}\left(x_{j}-x\right)^{m+1} N_{j}^{\rho}(x)\right)
$$

La somme en $j$ s'étend pour les indices des particules dont $x$ appartient au support de $N_{j}^{\rho}$, c'est-à-dire pour les particules $x_{j}$ telles que $N_{j}^{\rho}(x)$ n'est pas nul. Le nombre de termes dans cette somme dépend de $x$ et il est égal au nombre de particules « proches » de $x$. Soit $N_{\max }$ le nombre maximum de termes dans les différentes 
sommes. Le nombre $N_{\max }$ est tel que pour aucun point $x$ le nombre de particules « proches » excède $N_{\max }$. Or, dans la somme en $i$ on n'y trouve que les $p+1$ nœuds de l'élément où appartient $x$. On en déduit ainsi que (32) peut se réécrire

$$
\left|u(x)-\left(u^{h}+u^{\rho}\right)(x)\right| \leq \frac{1}{(m+1) !}\left|\frac{\mathrm{d}^{m+1} u(\xi)}{\mathrm{d} x^{m+1}}\right| \times\left(N_{\max } \rho^{m+1} \max _{i \in I^{\rho}}\left|N_{i}^{\rho}(x)\right|+(p+1) h^{m+1} \max _{i \in I^{h}}\left|N_{i}^{h}(x)\right|\right) .
$$

Étant donné que les fonctions $N_{j}^{\rho}$ sont bornées dans le résultat du Lemme 3.4, et que les fonctions de forme des éléments finis sont partout inférieures à 1, on peut déduire la borne suivante

$$
\left\|u-\left(u^{\rho}+u^{h}\right)\right\|_{L^{\infty}} \leq\left[C_{1} h^{m+1}+C_{2} \frac{h^{p+1}}{\rho^{p+1}} \rho^{m+1}\right]\left\|\frac{\mathrm{d}^{m+1} u(\xi)}{\mathrm{d} x^{m+1}}\right\|_{L^{\infty}}=h^{p+1}\left[C_{1} h^{q}+C_{2} \rho^{q}\right]\left\|\frac{\mathrm{d}^{m+1} u(\xi)}{\mathrm{d} x^{m+1}}\right\|_{L^{\infty}}
$$

où $C_{1}$ et $C_{2}$ sont des constantes indépendantes de $\rho$ et $h$.

\section{EXEMPLES}

\subsection{Convergence de l'interpolation mixte}

Dans cette section on montre que les résultats de convergence qu'on a introduits dans la section précédente peuvent se reproduire par des expériences numériques. On en déduit, par voie de conséquence que les bornes qu'on a trouvées sont optimales.

On va donc interpoler une fonction simple en utilisant des éléments finis et des particules distribués uniformément dans le domaine. On prend comme exemple la fonction

$$
u(x)=x^{4}+2 x^{3}
$$

dans l'intervalle $\bar{\Omega}=[-1,1]$. On conserve la notation qu'on a utilisée jusqu'ici : $p$ est le degré d'interpolation des éléments finis, $m$ est l'ordre de consistance atteint avec les particules, $q:=m-p$ est le « gain de consistance », $h$ est la taille caractéristique des éléments finis et $\rho$ est la valeur du paramètre de dilatation des particules.

L'erreur se mesure avec la norme de $L^{2}(\Omega)$. Dans la figure 1 on représente pour différentes valeurs de $p$ et $q$ l'erreur en fonction du nombre de degrés de liberté lorsqu'on raffine en réduisant $h$ et $\rho$ au même temps, c'est-à-dire en gardant $h / \rho=$ cte. Rappelons que pour réduire $\rho$ il faut augmenter le nombre de particules. On remarque que l'erreur est $\mathcal{O}\left(h^{m+1}\right)$, c'est-à-dire, le même taux de convergence que l'on obtiendrait avec des éléments finis d'ordre $m$ ou avec EFG de consistance $m$

Dans la figure 2 on représente l'erreur obtenue en augmentant le nombre d'éléments tout en conservant la distribution des particules. Lorsque $\rho$ reste constant, l'erreur est $\mathcal{O}\left(h^{p+1}\right)$ si $\rho$ est assez grand (on obtiendrait le même s'il n'y avait pas de particules). Cependant, si $\rho$ est assez petit (avec le nombre de particules suffisant) on observe que l'erreur est $\mathcal{O}\left(h^{m+1}\right)$, c'est-à-dire que le taux de convergence s'améliore et en raffinant des éléments finis de degré $p$ on obtient le même comportement que s'ils étaient de degré $m$. Pour ceci, il faut que le nuage de particules soit assez dense pour considérer qu'il a permis d'enrichir la consistance de l'interpolation partout.

Dans la figure 3 on raffine seulement les particules et on garde le maillage d'éléments finis constant. Lorsque $h$ est assez petit on obtient l'erreur $\mathcal{O}\left(\rho^{q}\right)$, mais lorsque $h$ n'est plus si petit on ne retrouve plus la convergence puisque l'erreur ne diminue point en augmentant le nombre de particules.

Remarque 4.1. Cette analyse est également valable pour des fonctions qui ne sont pas polynômiques. En répétant l'expérience avec une fonction beaucoup plus complexe, par exemple

$$
u(x)=\sin \left(\frac{7}{6} \pi(x+1)\right) \cos ^{3}\left(\frac{35}{6} \pi(x+1)\right)
$$

dont la courbe représentative se trouve dans la figure 4, les résultats sont identiques. 

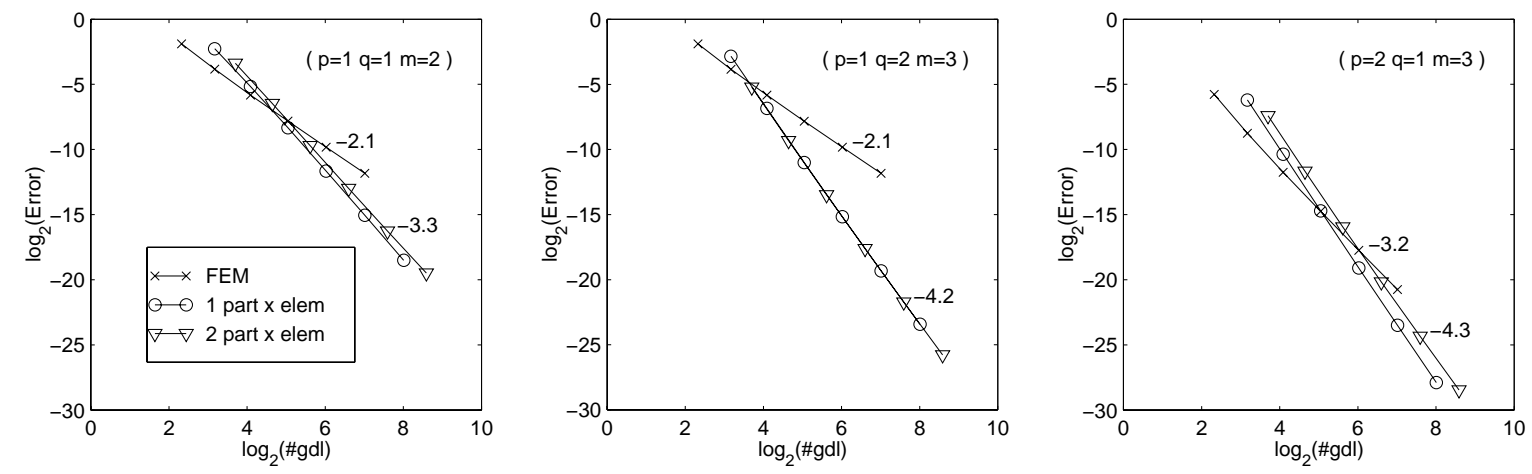

Figure 1. Convergence lorsqu'on raffine les nouds et les particules : $h / \rho$ constante et $h \rightarrow 0$. [Convergence for a mesh and meshless refinement: constant $h / \rho$ and $h \rightarrow 0$ ].
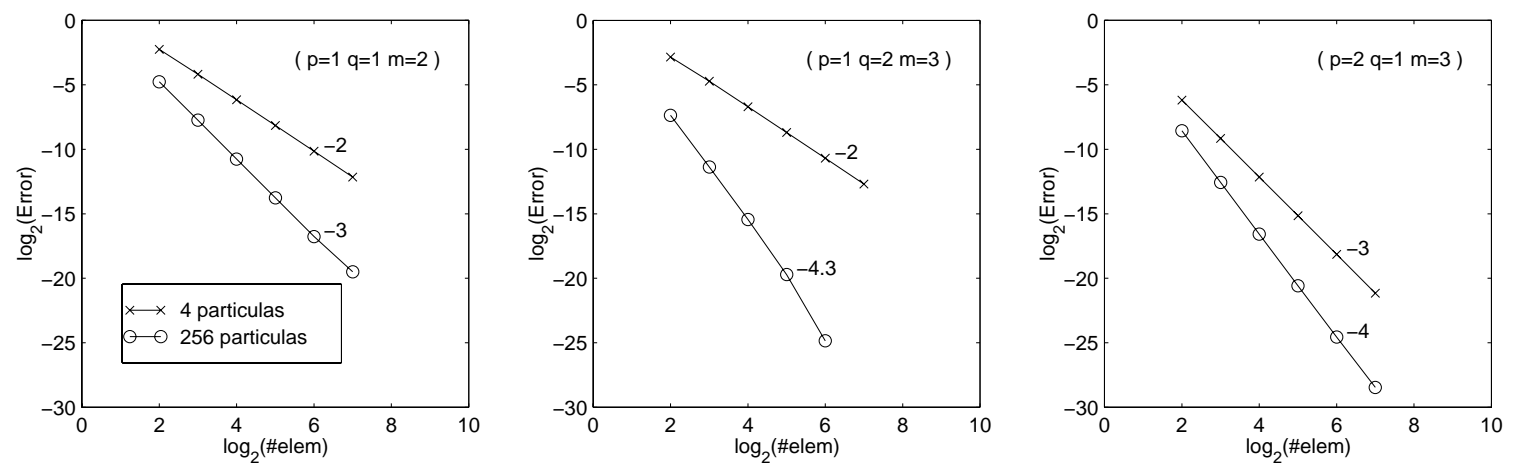

Figure 2. Convergence lorsqu'on ne raffine que les nœuds $: \rho$ constante et $h \rightarrow 0$.

[Convergence for a mesh refinement: constant $\rho$ and $h \rightarrow 0$ ].
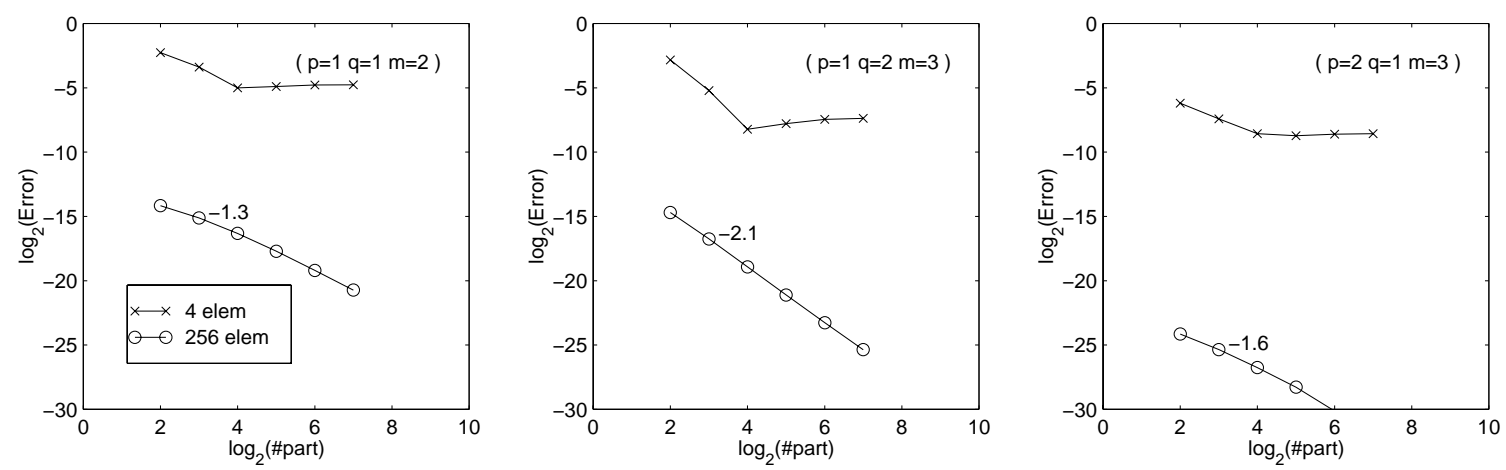

Figure 3. Convergence lorsqu'on ne raffine que les particules : $h$ constante et $\rho \rightarrow 0$.

[Convergence for a meshless refinement: constant $h$ and $\rho \rightarrow 0$ ]. 


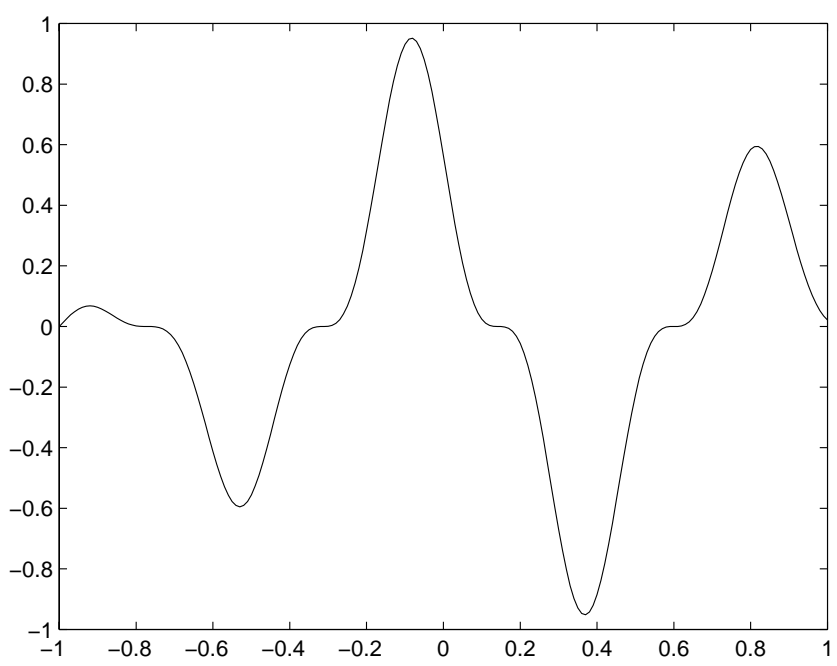

Figure 4. Courbe représentant la fonction définie dans (33). [Function $u(x)$ defined in (33)].

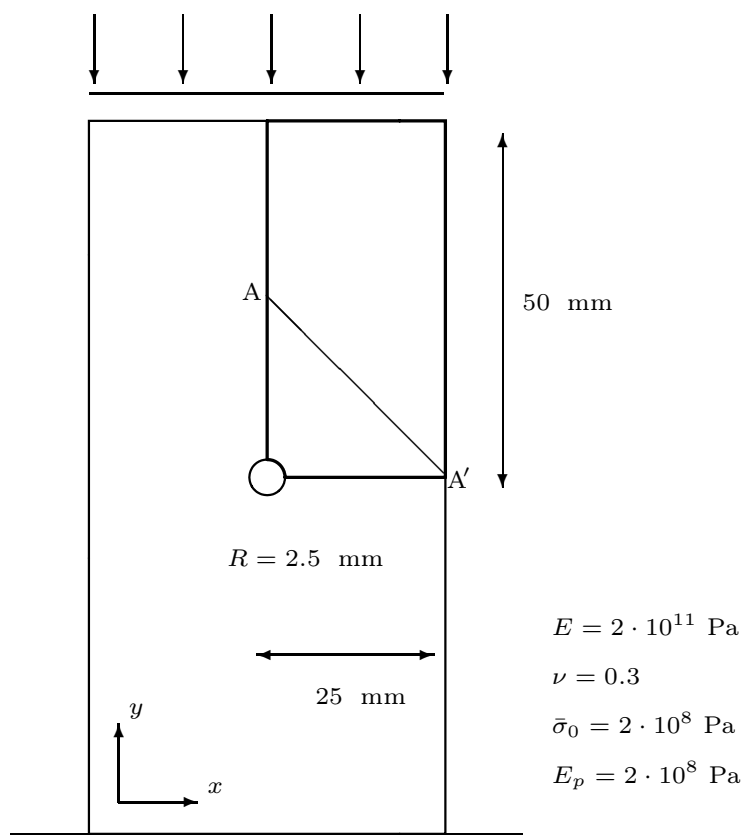

Figure 5. Présentation du problème : éprouvette rectangulaire avec une imperfection au centre. [Problem statement: rectangular specimen with one centred imperfection].

\subsection{Enrichissement dans un calcul non linéaire}

Cet exemple montre l'application de l'enrichissement des éléments finis par des méthodes de particules dans le cadre d'un problème non linéaire. Une éprouvette rectangulaire avec une imperfection est soumise à un chargement vertical, voir [7,9]. Le problème a deux axes de symétrie et on suppose un modèle avec un matériau élastoplastique bilinéaire et des conditions de déformation plane. La géométrie du problème ainsi que les propriétés du matériau sont présentées dans la figure 5. 

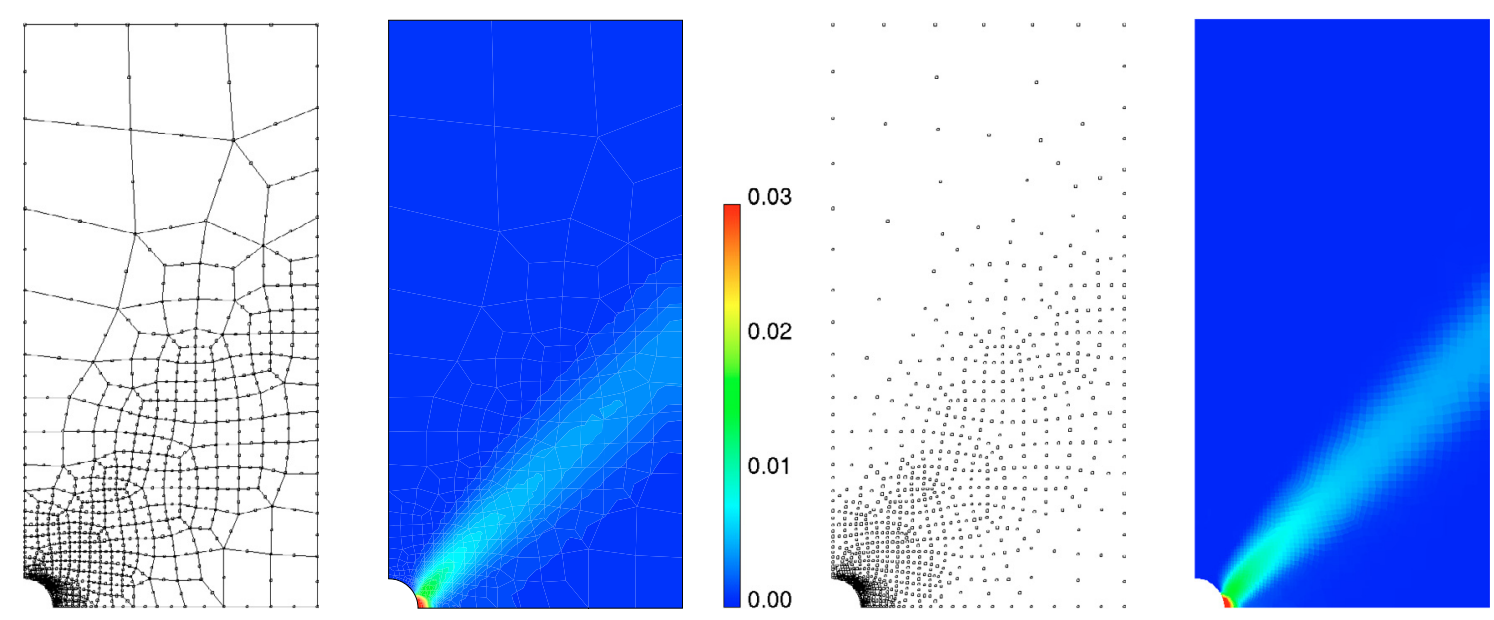

Figure 6. Maillage final et distribution de déformation inélastique équivalente associée pour un calcul standard par éléments finis de 8 nœuds (à gauche) et distribution de particules et la déformation inélastique équivalente calculée avec EFG (à droite).

[Final mesh with its corresponding equivalent inelastic strain for a standard finite element (8 noded elements) computation (left) and distribution of particles with its inelastic strain distribution for EFG (right)].

Ce problème a été résolu avec des éléments quadrangulaires de huit nœuds en utilisant une méthode de remaillage adaptatif pour obtenir une solution avec une précision satisfaisante [9,10]. La partie gauche de la figure 6 montre le maillage final obtenu par cette procédure de remaillage, ainsi que la distribution de déformation inélastique équivalente. Ce maillage a 2022 degrés de liberté et une erreur relative (en norme énergétique) de $0,18 \%$.

Cet exemple a été aussi résolu par la méthode «Element Free Galerkin ». Afin d'obtenir des résultats comparables, la distribution de particules utilisée coïncide avec la distribution de nœuds dans le maillage d'éléments finis et on a demandé de la consistance d'ordre deux. Le nombre de degrés de liberté est donc le même, 2022. Dans la partie droite de la figure 6 on trouve la distribution de particules et des déformations inélastiques équivalentes.

Néanmoins, bien que les résultats précédents aient une très bonne qualité, si on emploie un maillage plus grossier d'éléments finis de quatre nœuds (308 degrés de liberté) la solution obtenue devient beaucoup moins bonne, voir figure 7. On peut récupérer une solution aussi précise que la précédente et avec un nombre similaire de degrés de liberté en ajoutant des particules au maillage grossier. Effectivement, si on ajoute des particules jusqu'à atteindre $308+906=1214$ degrés de liberté et on augmente l'ordre de consistance $(m=2)$, la distribution des déformations inélastiques équivalentes obtenue récupère la netteté qu'on avait obtenu avec les premiers maillages, voir figure 7. Il faut remarquer que le maillage final de la procédure adaptive (Fig. 6, à gauche) a été obtenu après quelques itérations de remaillage, c'est-à-dire, en refaisant le maillage de zéro plusieurs fois. Dans le dernier exemple (Fig. 7) le maillage d'éléments finis est toujours le même et les particules s'ajoutent là où c'est nécessaire.

Dans la figure 8 on montre la distribution des déformations inélastiques équivalentes le long du segment $\left(\mathrm{A}-\mathrm{A}^{\prime}\right)$ pour chacune des discrétisations considérées. La définition du segment $\left(\mathrm{A}-\mathrm{A}^{\prime}\right)$ se trouve dans la figure 5.

\section{Conclusions}

On a présenté l'analyse de la convergence d'une interpolation mixte qui enrichit l'approximation par éléments finis avec des méthodes de particules. L'expression de la borne d'erreur nous indique quelles sont les stratégies 

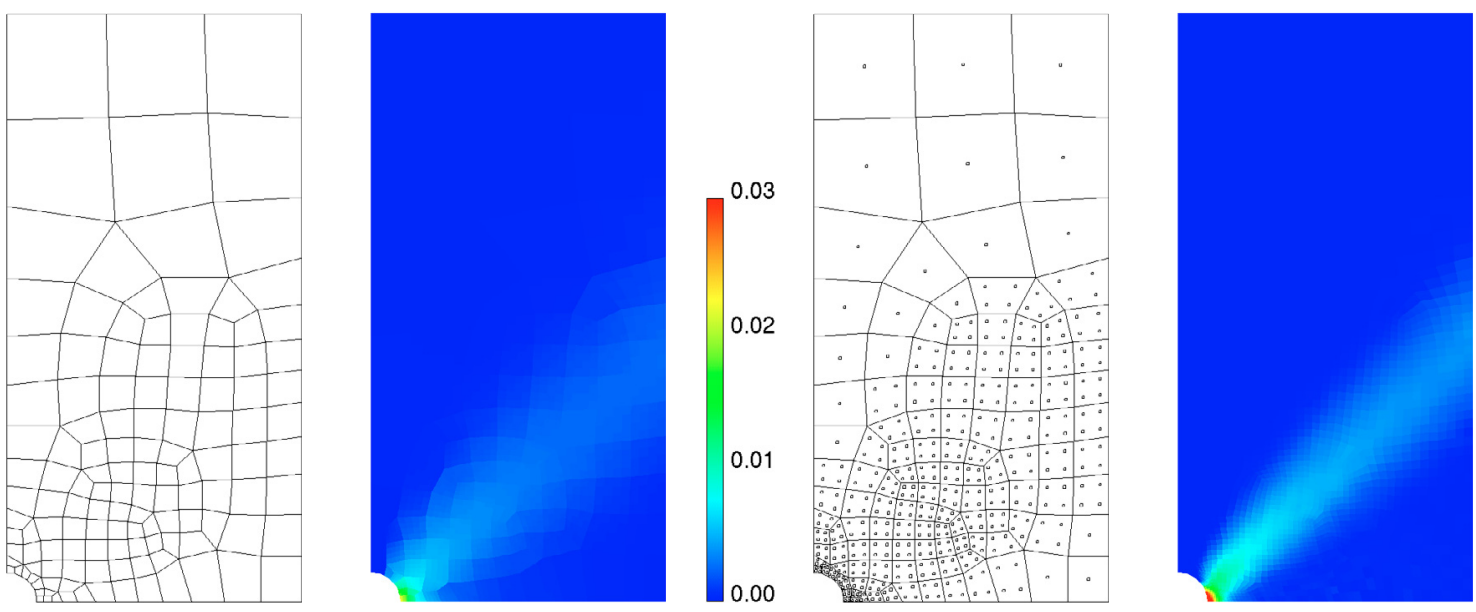

FiguRE 7. Maillage grossier (éléments Q1) avec la distribution des déformations inélastiques équivalentes correspondante (gauche) et interpolation mixte avec sa distribution des déformations inélastiques équivalentes (droite).

[Coarse finite element mesh (Q1 elements) with its corresponding equivalent inelastic strain (left) and mixed interpolation with its equivalent inelastic strain distribution (right)].
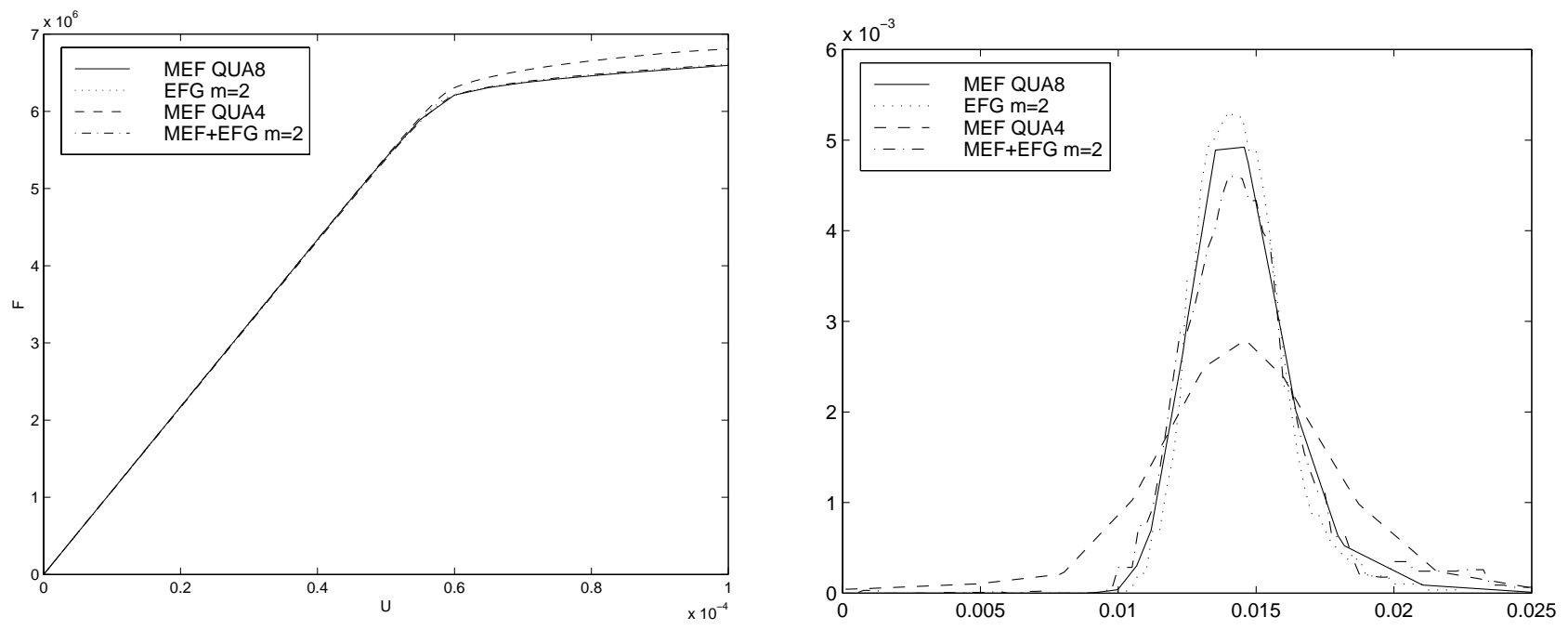

FigurE 8. Force versus déplacement (gauche) et déformations inélastiques le long du segment $\left(\mathrm{A}-\mathrm{A}^{\prime}\right)$ pour chaque discrétisation (droite).

[Force versus displacement (left) and evolution of the equivalent inelastic strain along $\left(\mathrm{A}-\mathrm{A}^{\prime}\right)$ for each approximation (right)].

de raffinement qui conduisent à une solution convergente. Le comportement de la méthode mixte établi par le résultat théorique se confirme par des résultats numériques.

\section{RÉFÉRENCES}

[1] T. Belytschko, Y.Y. Lu et L. Gu, Element-free Galerkin methods. Internat. J. Numer. Methods Engrg. 37 (1994) $229-256$. 
[2] T. Belytschko et D. Organ, Element-free Galerkin methods for dynamic fracture in concrete. D.R.J. Owen, E. Oñate and E. Hilton Eds., Comp. Plasticity. Fundamentals and Applications (1997) 304-321.

[3] T. Belytschko, D. Organ et Y. Krongauz, A coupled finite element-free Galerkin method. Comput. Mech. 17 (1995) $186-195$.

[4] T. Belytschko et M. Tabbara, Dynamic fracture using element-free Galerkin methods. Internat. J. Numer. Methods Engrg. 39 (1996) 923-938.

[5] P. Breitkopf, G. Touzot et P. Villon, Consistency approach and diffuse derivation in element-free methods based on moving least squares approximation. Comput. Assist. Mech. Eng. Sci. 5 (1998) 479-501.

[6] P. Breitkopf, A. Rassineux, G. Touzon et P. Villon, Explicit form and efficient computation of MLS shape functions and their derivatives. Internat. J. Numer. Methods Engrg. 48 (2000) 451-466.

[7] P. Díez, M. Arroyo et A. Huerta, Adaptivity based on error estimation for viscoplastic softening materials. Mechanics of Cohesive-Frictional Materials 5 (2000) 87-112.

[8] D. Hegen, Element-free Galerkin methods in combination with finite element approaches. Comput. Methods Appl. Mech. Engrg. 135 (1996) 143-166.

[9] A. Huerta et P. Díez, Error estimation including pollution assessment for nonlinear finite element analysis. Comput. Methods Appl. Mech. Engrg. 180 (2000) 21-41.

[10] A. Huerta, A. Rodríguez-Ferran, P. Díez et J. Sarrate, Adaptive finite element strategies based on error analysis. Internat. J. Numer. Meth. Engrg. 46 (1999) 1803-1818.

[11] A. Huerta et S. Fernández-Méndez, Enrichment and coupling of the finite element and meshless methods. Internat. J. Numer. Methods Engrg. 48 (2000) 1615-1636.

[12] S. Kulasegaram et J. Bonet, Corrected smooth particle hydrodynamics method for metal forming simulations. J. Huétink and F.P.T. Baaijens Eds., Simulation of Materials Processing: Theory, Methods and Applications (1998) 137-142.

[13] W.K. Liu et Y. Chen, Wavelet and multiple scale reproducing kernel methods. Internat. J. Numer. Methods Fluids 21 (1995) 901-931.

[14] W.K. Liu, S. Jun et Y.F. Zhang, Reproducing kernel particle methods. Internat. J. Numer. Methods Fluids 20 (1995) 10811106.

[15] W.K. Liu, S. Li et T. Belytschko, Moving least square reproducing kernel methods. (I) Methodology and convergence. Comput. Methods Appl. Mech. Engrg. 143 (1996) 113-154.

[16] W.K. Liu, R.A. Uras et Y. Chen, Enrichment of the finite element method with reproducing kernel particle method. J. Appl. Mech. 64 (1997) 861-870.

[17] Y.Y. Lu, T. Belytschko et L. Gu, A new implementation of the element free Galerkin method. Comput. Methods Appl. Mech. Engrg. 113 (1994) 397-414.

[18] B. Nayroles, G. Touzot et P. Villon, Generalizing the finite element method: diffuse approximation and diffuse elements. Comput. Mech. 10 (1992) 307-318.

[19] D. Organ, M. Fleming, T. Terry et T. Belytschko, Continuous meshless approximations for nonconvex bodies by diffraction and transparency. Comput. Mech. 18 (1996) 225-235.

[20] J.P. Vila, On particle weighted methods and smooth particle hydrodynamics. Math. Models Methods Appl. Sci. 9 (1999) 161-209.

[21] P. Villon, Contribution à l'optimisation. Thèse d'état, Université Technologique de Compiègne (1991). 